\title{
A spatial individual-based model predicting a great impact of copious sugar sources and resting sites on survival of Anopheles gambiae and malaria parasite transmission
}

\author{
Lin Zhu ${ }^{1 *}$, Whitney A Qualls ${ }^{1}$, John M Marshall ${ }^{2}$, Kris L Arheart ${ }^{1}$, Donald L DeAngelis ${ }^{3}$, John W McManus ${ }^{4}$,
} Sekou F Traore ${ }^{5}$, Seydou Doumbia ${ }^{5}$, Yosef Schlein ${ }^{6}$, Günter C Müller ${ }^{6}$ and John C Beier ${ }^{1}$

\begin{abstract}
Background: Agent-based modelling (ABM) has been used to simulate mosquito life cycles and to evaluate vector control applications. However, most models lack sugar-feeding and resting behaviours or are based on mathematical equations lacking individual level randomness and spatial components of mosquito life. Here, a spatial individual-based model (IBM) incorporating sugar-feeding and resting behaviours of the malaria vector Anopheles gambiae was developed to estimate the impact of environmental sugar sources and resting sites on survival and biting behaviour.

Methods: A spatial IBM containing An. gambiae mosquitoes and humans, as well as the village environment of houses, sugar sources, resting sites and larval habitat sites was developed. Anopheles gambiae behaviour rules were attributed at each step of the IBM: resting, host seeking, sugar feeding and breeding. Each step represented one second of time, and each simulation was set to run for 60 days and repeated 50 times. Scenarios of different densities and spatial distributions of sugar sources and outdoor resting sites were simulated and compared.

Results: When the number of natural sugar sources was increased from 0 to 100 while the number of resting sites was held constant, mean daily survival rate increased from $2.5 \%$ to $85.1 \%$ for males and from $2.5 \%$ to $94.5 \%$ for females, mean human biting rate increased from 0 to 0.94 bites per human per day, and mean daily abundance increased from 1 to 477 for males and from 1 to 1,428 for females. When the number of outdoor resting sites was increased from 0 to 50 while the number of sugar sources was held constant, mean daily survival rate increased from $77.3 \%$ to $84.3 \%$ for males and from $86.7 \%$ to $93.9 \%$ for females, mean human biting rate increased from 0 to 0.52 bites per human per day, and mean daily abundance increased from 62 to 349 for males and from 257 to 1120 for females. All increases were significant $(P<0.01)$. Survival was greater when sugar sources were randomly distributed in the whole village compared to clustering around outdoor resting sites or houses.

Conclusions: Increases in densities of sugar sources or outdoor resting sites significantly increase the survival and human biting rates of An. gambiae mosquitoes. Survival of An. gambiae is more supported by random distribution of sugar sources than clustering of sugar sources around resting sites or houses. Density and spatial distribution of natural sugar sources and outdoor resting sites modulate vector populations and human biting rates, and thus malaria parasite transmission.
\end{abstract}

Keywords: Malaria, Anopheles gambiae, Sugar-feeding, Resting, Behavior, Individual-based model, Agent-based model

\footnotetext{
*Correspondence: I.zhu3@med.miami.edu

'Department of Public Health Sciences, Miller School of Medicine, University of Miami, Miami, Florida, USA

Full list of author information is available at the end of the article
}

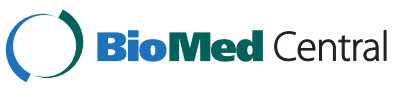

(c) 2015 Zhu et al.; licensee BioMed Central. This is an Open Access article distributed under the terms of the Creative Commons Attribution License (http://creativecommons.org/licenses/by/4.0), which permits unrestricted use, distribution, and reproduction in any medium, provided the original work is properly credited. The Creative Commons Public Domain Dedication waiver (http://creativecommons.org/publicdomain/zero/1.0/) applies to the data made available in this article, unless otherwise stated. 


\section{Background}

Malaria parasites are transmitted through the bites of anopheline mosquitoes, and the intensity of transmission largely depends on survival and human biting behaviour of the vector $[1,2]$. To survive, both male and female mosquitoes feed on sugar for energy [3]. Male mosquitoes depend exclusively on sugars for nutrients, while female mosquitoes feed on sugars for daily energy requirements, (e.g., flying, mating, etc.), and feed on blood for egg development, although blood could also be used as energy when sugar sources are completely unavailable [4]. Females need sugar which they require soon after emergence and sometimes also before blood feeding [3,5]. Optimum sugar feeding can prolong the mosquito lifespan and fecundity [6]. Population size and daily survival rates of anopheline mosquitoes are higher in natural sugar rich areas than in natural sugar poor areas $[7,8]$. Sugar source availability can also affect the sequence of behaviours after emergence $[9,10]$. A laboratory experiment showed that female Anopheles gambiae feed on sugar significantly more frequently in the absence of a blood source [11]. Conversely, as sugar and blood are energetically interchangeable, blood feeding frequency can increase when sugar sources are not available $[5,8,11,12]$, and disturb the timing for oviposition and gonotrophic cycle [5]. Accordingly, the reduction of sugar sources can reduce the survival and the abundance of anopheline mosquitoes, but increase blood seeking and feeding frequency of each female mosquito at the same time; therefore, the impact of natural sugar sources on malaria transmission remains unclear.

A major part of adult mosquito life is spent in resting sites $[13,14]$. While different species have various diel activity patterns, anopheline mosquitoes mainly rest during the daytime and complete activities like sugar feeding and blood feeding during the night, and then return to resting sites [13]. Difficulty in finding a suitable resting site can result in additional flight time, which consumes more energy, leading to increased demand of sugar intake. Thus, availability of resting sites may affect their sugar-feeding behaviour and survival. In addition, if mosquitoes cannot find proper resting sites, they have a higher likelihood of being exposed to heat and sunlight, thereby increasing dehydration and mortality rate [15].

Readily available sugar sources and resting sites promote the survival and biting behaviour of anopheline mosquitoes, and as a byproduct they also affect their ability to transmit malaria parasites. However, there is limited research that addresses sugar-feeding and resting behaviours compared to blood feeding behaviour. The reason could be that sugar-feeding and resting behaviours are not directly associated with malaria transmission, which provokes less interest and limited research funding [16]. In addition, tracking sugar-feeding and resting behaviours of mosquitoes in field studies can be very difficult and have problems such as ethical issues [16]. With information from studies on the sugar-feeding and resting habits of malaria vectors, agent-based modelling $(\mathrm{ABM}) /$ individual-based modelling (IBM) can be an exceptionally suitable tool for predicting the outcome of given situations on anopheline survival by including these factors and simulating the interactions between the mosquitoes and their environment of sugar sources, resting sites, larval habitat sites, houses, and humans.

Several ABMs have been developed to simulate mosquito life cycles and their interactions with humans, and to evaluate vector control applications of larval source management (LSM), long-lasting insecticidal nets (LLINs) and indoor residual spraying (IRS) [17-21]. However, only few modelling studies included sugar feeding and resting behaviours. One previous mathematical modelling study incorporated sugar feeding of mosquitoes and estimated the daily sugar feeding rates at a field site in Mali [22]. It also examined the potential effectiveness of combining attractive toxic sugar baits (ATSB) [23] targeting the sugarfeeding behaviour with other vector control methods, such as LLINs and IRS [22]. However, this and other models lack spatial components (locations of objects, distances between objects, etc.), which limit the ability to estimate the impact of spatial configuration of objects in the environment. Another study developed a dynamic state variable model to predict the decisions of female mosquitoes selecting different behaviours including sugarfeeding, blood-feeding and ovipositing based on the physiological state and location of the mosquito. However, it emphasized the behaviour decisions and the location was only identified as indoors or outdoors [24]. In addition, current IBMs have simulated the interactions with time-step resolutions at hourly or even daily intervals [20], assuming that mosquitoes remain in the same state and perform only one behaviour in a whole hour or day, which is not realistic. Also, in current spatial IBMs, landscapes were set to be composed of a course grid of a small number of grid cells (e.g. 40 grid cells [17]), so each grid cell represents a large area. During each step mosquitoes would move a whole grid cell, reducing the amount of realistic stochasticity in the mosquito movement. The host seeking functions in the models also assumed that the mosquitoes could always find the resources in the eight adjacent grids, no matter what the target was and how much area one grid cell represented $[17,20]$. However, the attractiveness or attractive ranges of different resources/ targets can be very different [25-27]. Hence, the success rate of resource-seeking, including human host-seeking, in current IBMs can be biased, leading to inaccurate estimation of human biting and malaria transmission. Further, human hosts in current IBMs are always static (representing humans sleeping in houses) in assigned grids $[17,20]$. 
However, with the use of vector control LLINs and IRS, indoor biting has been demonstrated to shift from indoors to outdoors $[28,29]$. Thus, if human hosts remain static indoors, there can be a decrease in host availability to mosquitoes that are far from house locations; this obviously leads to an underestimation of outdoor human biting rates, and also leads to female mosquitoes more concentrated around houses.

To estimate the impact of environmental sugar sources and outdoor resting sites on the survival and human biting rate of An. gambiae, a spatial IBM was developed that takes into consideration the sugar-feeding and resting behaviours, and aforementioned simplifications assumed in other models. The IBM used a continuous landscape, a time-step resolution of one second, specific attractive distances of different objects, moving human hosts, and An. gambiae with functions of sugar-feeding and resting behaviours. This model is the first IBM to examine both sugar-feeding and resting behaviours in the mosquito life cycle, and consider the potential impact of environmental structure of sugar sources and resting sites on malaria transmission. This study provides a basis for evaluating new vector control interventions targeting sugar feeding or resting behaviours.

\section{Methods}

The ODD (Overview, Design concepts, and Details) protocol was developed in 2006 [30] and revised in 2010 [31] to standardize the description of ABM/IBM in publications. It helps to make the $\mathrm{ABM} / \mathrm{IBM}$ more understandable, complete, and reproducible. The ODD protocol [31] is used here to describe the model.

\section{Purpose}

The purpose of the model is to estimate the impact of the density and configuration of environmental sugar sources and outdoor resting sites on the survival and human biting rate of An. gambiae in a village setting.

\section{Entities, state variables, and scales Entities \\ Living entities}

The living entities consisted of two types of agents: humans and An. gambiae mosquitoes.

Humans A total of 60 humans were randomly assigned to the 20 houses in the center of the village and all assignments remained stable during the course of each repetition for the simulations.

Mosquitoes The population of An. gambiae mosquitoes, males and females individually, were simulated over their individual lifetimes. The number of living mosquitoes could change depending on birth and death rates through time.

\section{Non-living entities}

The non-living entities consisted of a village landscape, houses, outdoor resting sites, sugar sources and larval habitat sites.

Landscape A continuous two-dimensional space with a reflecting boundary was used to simulate the landscape. This allowed the moving agents (mosquitoes or humans) that would hit one of the boundaries to be reflected back instead of being permanently removed (absorbing boundary) or entering from the opposite side (non-absorbing boundary). Reflecting boundaries are considered more realistic for this study because Anopheles mosquitoes do not usually fly too far from their breeding habitats [32], and people in the village who may reach/move out of the village boundary are more likely to return from the same boundary.

Village and houses Because the maximum flight distance of An. gambiae is estimated to be 200 to 400 metres [33], also in accordance with the common size of a village in Mali, the area of the village in the IBM was set to be $600 \times 600$ metres. In accordance with geographic configuration in Mali, the 20 houses were set to be randomly located in the center of the village in a grid of $100 \times 100$ metres. Once the location of each house was determined, it remained the same through all the simulation scenarios and repetitions.

Outdoor resting sites A number of natural outdoor resting sites were used, and the locations were randomly selected and constant throughout all repetitions of each simulation, though densities of resting sites could differ between simulations. Houses were also considered to be resting sites but not counted in the number of outdoor resting sites.

Sugar sources A number of sugar sources were used and scattered randomly throughout the area in simulation scenarios of different sugar source densities and scenarios of random distribution of sugar sources. In other scenarios, distributions adjacent to outdoor resting sites or houses, sugar sources were placed at the same locations of resting sites or houses. The numbers and locations remain constant throughout all repetitions of each simulation, but could change from simulation to simulation.

Larval habitat sites Fifty larval habitat sites were scattered randomly throughout the area and remained constant throughout all simulations. 


\section{State variables}

Each mosquito was characterized by variables for age (number of time steps since emergence), sugar (numbers representing the extent a mosquito needs sugar meals), blood (in females), and gravid status (in females, numbers representing pre-gravid, and length in days being gravid), as well as its locations (coordinates) in space: at a resting site, at a sugar source, at a larval habitat site (in females), on a human (in females), or in targeted or random movement. These variables represent their states/status in each step. Each human was characterized by variables for bites and their location in space. Each larval habitat site was characterized by variables for eggs (number of eggs oviposited in each aquatic site per day) and its location in space. All other agents have variables of locations in space.

\section{Temporal scales}

The time-step resolution was one second, meaning that each step represented one second. Simulations were performed over periods of 60 days.

\section{Process overview and scheduling Humans}

Once homes were assigned to humans, they remained the same through each repetition of simulations. The humans had functions of random movement and targeted movement: beginning at 07:00 each day, they moved out of their homes in random directions at each step; beginning at 20:00, they moved back, targeting their assigned homes until they arrived; then they remained at home during the night. When a human got a bite from a female An. gambiae, the bite counter would increase by 1 .

\section{Anopheles gambiae}

The life cycles of an adult An. gambiae simulated in the model is shown in Figure 1. An average of 7 days was used for the longest life span for males [34]. From day 2, each individual male An. gambiae looped between behaviours until mortality was recorded. An estimate of 21 days was used for the longest life span for females [34]. Successful mating of every female was assumed. For females needing a blood meal, if it was not available during a 5 hour time span at night, the mosquito would switch to sugar-seeking [35]. Variable "sugar" records changes in need of sugar meal, and triggers sugar source seeking. Males needed at least two sugar meals per night while females did not have a minimal sugar meal requirement if they could get blood meals [36]. However, for both males and females, every additional flight of 2,000 steps, which generally represented 2000 metres, was assumed to lead to an additional need of one sugar meal [4]. At the beginning of each day, variable "sugar" of every mosquito will increase by one (need for one sugar meal) to count for energy consumption while resting. Mosquitoes that were not able to find an energy resource for a whole night would die of starvation. Blood-fed females would need two to three days to be ready to oviposit [37]. The number of eggs produced by gravid female $A n$. gambiae was 100, variations due to individual physiological fitness and different blood meal size were not considered [38]. The development of their aquatic stages is described in the recruitment submodel section below.

\section{Sequence of actions}

The actions of each agent were considered sequentially. The agent being considered is called the 'active' agent, while any agent that is being acted on by the active agent is called the 'passive' agent. In each step, each active agent began by checking its own status, and then the desired movement type was decided based on the status of the agent. After that, the active agents would check the surrounding environment, and make spatial movement based on the environment and movement rules described earlier in the $A n$. gambiae and human section. Finally the state variables of the agent would be updated as the result of the movement. If an interaction with another agent (usually a passive agent) occurred, a signal would also be sent to change the status of the other agent. The passive agents, on the other hand, would receive the signal from the interaction and change its state variable values accordingly. For example, in one step, age, sugar, blood, and gravid status of a female An. gambiae would be checked by the model, as well as the time of day; then, using that information, the model would decide if that female would seek a sugar source/blood source/larval habitat site/resting site or stay static. If the female mosquito needed a blood meal, then she would search the surrounding environment (usually a circle with a radius of the attractive distance of human) and see if there was a human within range. If there was then the female mosquito would make a targeted movement of one meter toward the human. With its movement type variable marked as targeted, she would continue to fly toward the human in the following steps without checking her status to decide desired movement, until it reached the human. If the blood-feeding interaction occurred, the model would change the value of the blood variable of the mosquito, and the human would receive a signal to increase the number of bites variable by 1 . In each step, the actions of humans were simulated before those of mosquitoes, and the order within humans and mosquitoes was random. When the step reached 19:00 of each day, an action of recruiting new mosquitoes would follow actions of humans and mosquitoes. 


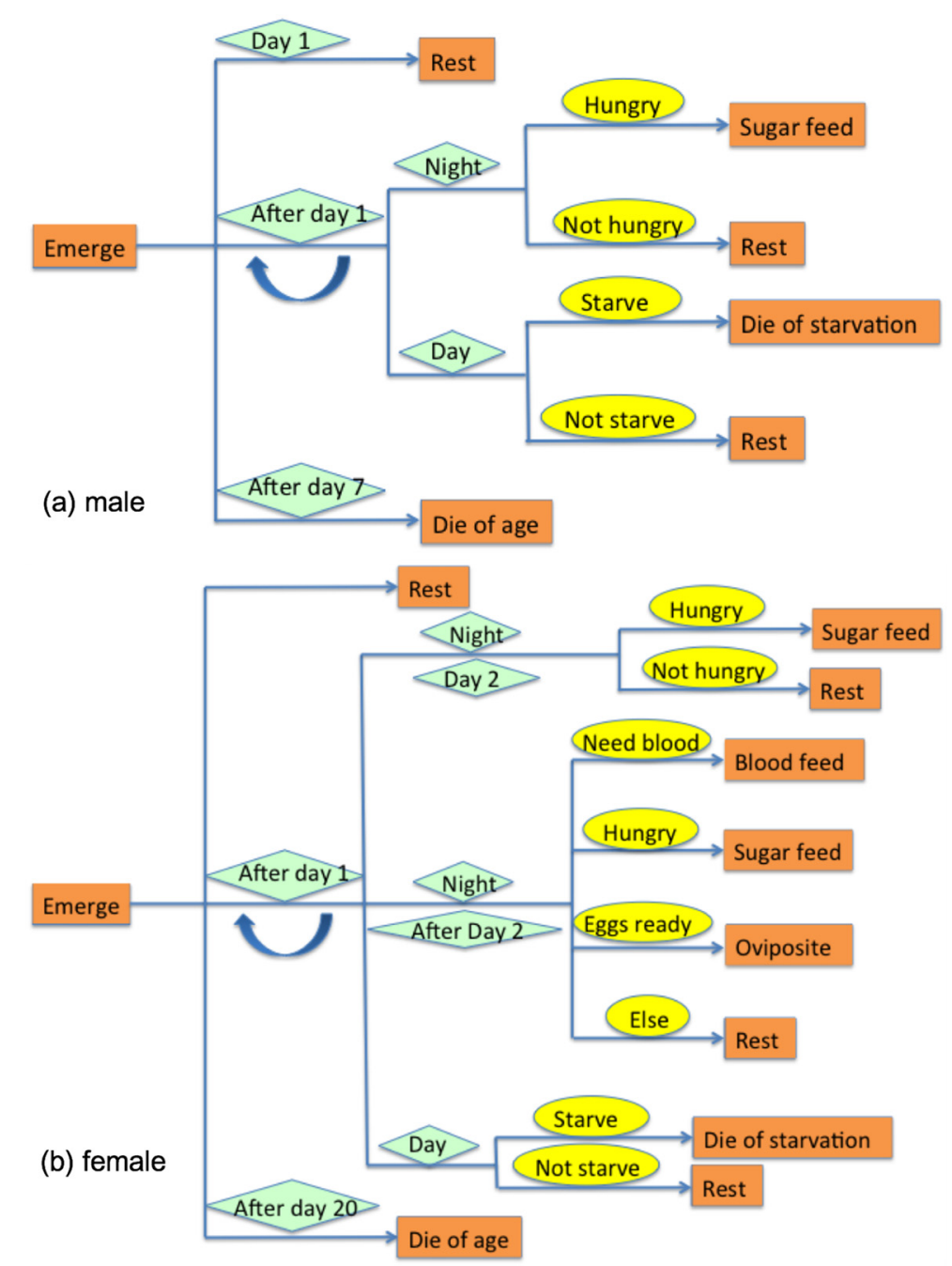

Figure 1 Life cycle of An. gambiae. Subfigures (a) and (b) represent life cycles of male An. gambiae and female An. gambiae. Orange rectangles represent the behaviours of the individuals, green diamonds represent the timing conditions, and the yellow ovals represent the status of the individuals. In each step, each An. gambiae would check its status and select a route in the figure.

\section{Time}

The beginning of a simulation was set to be 19:00 on day 1. Night was defined as 19:00 to 05:00, and daytime was defined as 05:00 to 19:00. Time of day was a determinant of the movement rules. Mosquitoes rested during daytime and flew during night. Humans were set to move out of their homes at 07:00 and return home at 20:00. New adult mosquitoes emerged at 19:00 (discussed in Recruitment submodel section).

\section{Design concepts}

\section{Basic principles}

The theories of sugar feeding, blood feeding, resting, and oviposition of An. gambiae mosquitoes are discussed in the Process Overview and Scheduling section. The model simulated all these behaviours and their interactions with the environment within a two-dimensional village setting.
The theories and hypotheses were also used in the submodels described below. The simulations were set to represent real-world scenarios and thus could be used to predict the impact of environmental sugar sources and outdoor resting sites on the survival and human biting behaviour of An. gambiae.

\section{Emergence}

The emergent output of most interest were the effects of numbers and spatial distributions of natural resting sites and sugar sources on the survival and human biting rates of An. gambiae mosquitoes, which vary in unpredictable ways as the environmental configuration changes.

\section{Adaptation}

The mosquito agents could make some simple adaptive decisions. As per the sample described in Sequence of 
Actions in the Process Overview and Scheduling section states, in one step, a female An. gambiae, according to it status, could decide to seek a resource or stay static. If the female mosquito was in need of a blood meal, then she would search the surrounding environment and see if there was a human within range. If there was then the female mosquito would move one meter toward the human, and continue to fly toward the human in the following steps until she reached the human. If the human was moving, then the mosquito would target the new location of the human in each step.

\section{Objectives}

The objectives calculated by the model were the daily abundances of mosquitoes and human biting rates. The adaptation traits of the individuals did not increase their success at meeting the objectives. The objectives were measured by recording the number of mosquitoes and total number of bites every day.

\section{Learning}

No learning behaviour by individuals was built into the model.

\section{Prediction}

The mosquito agents could make simple predictions about the location of humans, sugar sources, resting sites, and larval habitat sites from sensory input.

\section{Sensing}

The mosquitoes could sense humans, sugar sources, resting sites, and larval habitat sites within certain radiuses and move in the direction of the targets.

\section{Interaction}

The mosquitoes had interactions with humans through blood feeding, with sugar sources for sugar feeding, with resting sites for resting, and with larval habitat sites for oviposition. The blood feeding interaction affected the bite counter of the humans, and the ovipositing interaction affected the egg count of the larval habitat sites.

\section{Stochasticity}

The outdoor resting sites and sugar sources were assigned randomly for each simulation. Humans moved randomly outside of their houses. Mosquito movement was partly random, but partly directed when a target was detected.

\section{Collectives}

No intermediate collectives were considered.

\section{Observation}

Daily abundances of mosquitoes were recorded before and after recruitment each day. Daily number of bites was recorded at every blood-feeding event. Egg count was recorded for each larval habitat site at every oviposition event.

\section{Initialization}

Different scenarios were simulated to test the impact of sugar sources and outdoor resting sites (Figure 2), and each scenario was repeated 50 times. A total of 1,000 male and 1,000 female adult An. gambiae mosquitoes were released at the beginning of each simulation. The age, sugar, blood, gravid status, and location variables of each mosquito were randomly assigned and could differ between each repetition and simulation.

Location and a home were randomly assigned for each human at the beginning of simulation and could differ between each repetition and simulation. Fifty randomly located larval habitat sites were used through all simulation scenarios and repetitions.

Scenarios were initiated with different numbers of outdoor resting sites and sugar sources.

\section{Sugar sources}

To estimate the impact of different densities of sugar sources, a fixed number of 50 outdoor resting sites were used, and the locations were randomly selected and constant throughout all the simulations and repetitions of different densities of sugar sources. Sugar sources were simulated at six scenarios $0,5,25,50,75$ and 100 . The locations of sugar sources were randomly selected for each scenario and kept constant through repetitions.

\section{Outdoor resting sites}

To estimate the impact of different densities of outdoor resting sites, a fixed number of 25 sugar sources were used, and $0,5,10,20,30,40$ and 50 outdoor resting sites were tested as six scenarios. The randomness and repetitions of these simulations were similar to the sugar source simulations described above.

To estimate the impact of closeness between outdoor resting sites and sugar sources, 25 sugar sources and 25 outdoor resting sites at the same or random locations were simulated.

To estimate the impact of closeness between sugar sources and houses, one sugar source by each of the 20 houses with the other 5 randomly located and 25 randomly located sugar sources were simulated, and 50 outdoor resting sites were randomly located in both simulations.

\section{Input data}

The model does not use external input data to represent time-varying processes. 


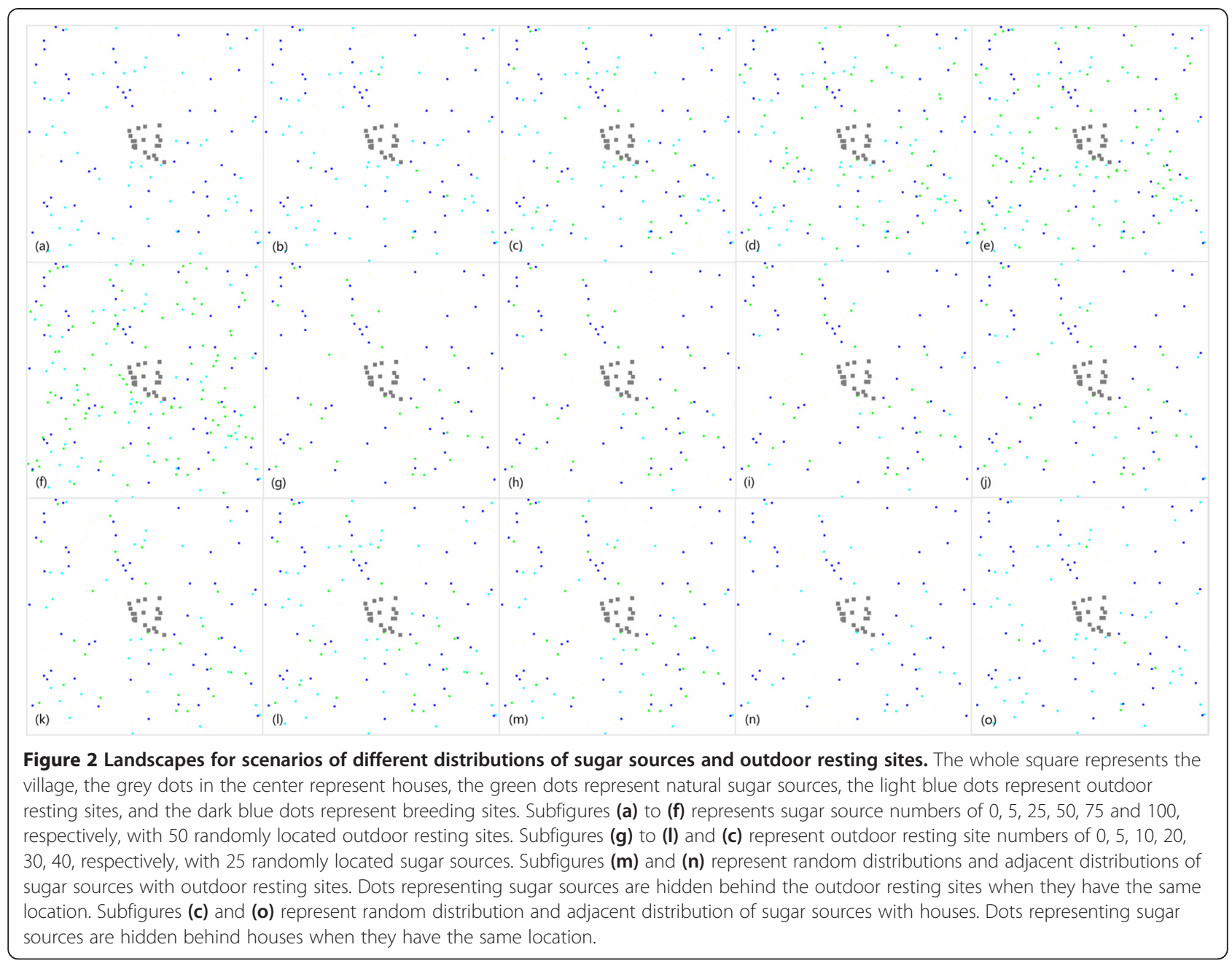

\section{Submodels}

\section{Resource-seeking submodel}

When an An. gambiae decided to sugar feed, blood feed, oviposit or rest, it would begin a resource-seeking procedure for the targeted object. The attractive distances of sugar sources, humans, larval habitat sites and resting sites were set to be 5 metres, 40 metres, 5 metres and 5 metres, respectively, according to the preliminary field study results (Günter C. Müller, unpublished data). Anopheles gambiae would search its surrounding environment within the radius of the attractive distance of its target. If there was no target in the circle, it would move randomly to one of the eight adjacent one-meter grid cells. If there was one target in the circle, it would move one grid cell towards the target and continue to move towards it in the following steps. If there were more than one target in the circle, it would first select one target randomly, and then move toward the target. Random selection was believed to be more realistic than selecting the nearest one because in front of many targets, mosquitoes always have a preference. For example, humans with higher $\mathrm{CO}_{2}$ output are more attractive [39]. The randomly selected target was assumed as the preferred target. Also, successful sugar feeding, blood feeding, ovipositing and resting were always assumed if An. gambiae arrived at the same location of the target.

\section{Recruitment submodel}

New adult An. gambiae would be recruited and emerge at 19:00 every day. Although An. gambiae mosquitoes actually emerge throughout the night instead of at one time point [40], they normally rest during the first night [41], so the assumption didn't affect the magnitude of results. However, the mosquito abundance would change sharply instead of smoothly because of this simplification. As the development time of eggs leading to adult emergence is about 12 days [40,42], for each of the first 12 days, there were no data on the oviposited egg counts 12 days before (simulation not started), so the number of recruited mosquitoes was assumed to equal the number of deaths of the prior day to achieve a steady state 
equilibrium population. From day 13, the number of recruited mosquitoes was a function of the total number of eggs oviposited 12 days before. Density-dependent development of the aquatic stages was accounted for in the function by setting a maximum egg capacity of each breeding site as 400 . This number was assumed because further details of type, area, predator, etc. of each breeding site was not included in this model. When the average number of eggs of the ovipositing day (12 days before the recruiting day), one day before, and one day after the ovipositing day exceeded the maximum egg capacity (400), then only 400 eggs would be counted as the effective egg number. When the average number of eggs was smaller than the maximum egg capacity, then the number of eggs of the ovipositing day would be used. Accounting for environmental factors such as drying of temporary breeding sites and the competition of the different aquatic stages of An. gambiae, 5\% of the eggs were assumed to develop to adult An. gambiae [40]. Equal numbers of new males and females were assumed. For example, if the average number of total oviposited eggs at one site at day 3, day 4 and day 5 exceeded 400, then only 400 would be counted as the number of effective eggs oviposited at day 4 , and the number of recruits of day 16 (12 days after day 4) would be $5 \%$ of 400 which is 20 ; if average number of total oviposited eggs at day 3, day 4 and day 5 was smaller than 400 , then the number of recruits of day 16 would be $5 \%$ of the number of oviposited eggs at day 4 . Half of the recruited mosquitoes would be males, and the other half would be females.
A summary of input parameters was provided in Table 1.

\section{Program}

JAVA 7 (Oracle Co., Redwood, CA) and Mason package v17 [43] were used to develop this model.

\section{Statistical analysis}

Daily survival rate was defined as the number of $A n$. gambiae mosquitoes at the end of day (before recruits) divided by the number at the beginning of the day (right after recruits). Human biting rate was defined as the total number of bites per day divided by the number of humans. Daily abundance was defined as the number of An. gambiae mosquitoes at the end of each day. Daily survival rates and abundances were calculated separately for males and females.

Because in the first 12 days, the number of deaths of the former day instead of the function of the number of eggs oviposited 12 days before was used to calculate the number of recruits, simulation was not realistic for the first 12 days. Hence only data from day 13 on were used for the analysis. The mean daily survival rate, human biting rate and daily abundance were calculated for each scenario; records from day 13 to day 60 for all 50 repetitions were used for the calculation. For comparison of average daily survival rates, human biting rates and abundances between scenarios, average area under curve (AUC) of the 48 days used was calculated for each repetition of simulation. ANOVA was used for the comparison, and the Tukey post hoc test was used to compare

Table 1 Parameter input used in the model

\begin{tabular}{|c|c|c|}
\hline Input/parameter & Value & Reference \\
\hline Human moving outdoors & 07:00 to $20: 00$ & Assumption \\
\hline Active time of An. gambiae & 19:00 to $05: 00$ & Assumption \\
\hline Life span of male An. gambiae & 7 days & [34] \\
\hline Life span of female An. gambiae & 21 days & {$[34]$} \\
\hline Threshold of blood-seeking female switching to sugar-seeking & 5 hours & [35] and assumption \\
\hline Minimum number of sugar meal of female An. gambiae per night & 2 & {$[36]$} \\
\hline Minimum number of sugar/blood meal of male An. gambiae per night & 1 & {$[36]$} \\
\hline Days needed to develop eggs after blood-feeding & $2 \sim 3$ days & {$[37]$} \\
\hline Average size of egg batches & 100 & {$[38]$} \\
\hline Attractive distance of sugar source & $5 \mathrm{~m}$ & Unpublished study results \\
\hline Attractive distance of human & $40 \mathrm{~m}$ & Unpublished study results \\
\hline Sensing distance of larval habitat site & $5 \mathrm{~m}$ & Unpublished study results \\
\hline Sensing distance of resting site & $5 \mathrm{~m}$ & Unpublished study results \\
\hline Days of aquatic cycle & 12 days & {$[40,42]$} \\
\hline Egg capacity of breeding site & 400 & Assumption \\
\hline Percentage of eggs developing to adults & $5 \%$ & {$[40]$} \\
\hline
\end{tabular}


between each density level of sugar sources and outdoor resting sites.

Generalized mixed linear regression model was used to control potential influence of time. Scenario, time (days) and their interaction term was included in the models. Time (days) was used as a repeated variable, and repetition within each scenario was used as subject variable. Either a first-order autoregressive structure, a compound-symmetry structure, or a variance components structure was used as the covariance structure, depending on which structure gave the best fit (smallest AIC). F test was used to examine the significance of each term. Least square means of each outcome variable were compared between scenarios using $t$ tests. A scenario of five sugar sources or outdoor resting sites was used to replace the scenario of 0 sugar sources or outdoor resting sites to improve the model fit.

SAS 9.3 (SAS Institute, Inc., Cary, NC) was used for the analyses.

\section{Results}

Table 2 shows the means and standard deviations of daily survival rates, human biting rates and daily abundances of different scenarios. Survival and human biting rates of An. gambiae increased with the increase of sugar source density and resting site density, even the densities increased by only 5 from 0 , the survival and human biting increased substantially. Daily abundances were higher when sugar sources were randomly distributed from outdoor resting sites, and both daily abundances and human biting rates were higher when sugar sources were randomly distributed from houses. Figure 3 shows the variation of survival and human biting rate along time with different sugar source or resting site densities. The order of magnitude of the five outcomes remains the same through time.

ANOVA of average AUCs shows that both the impacts of increasing the sugar source density and resting site density were substantial (Table 3 ). According to post hoc analysis, daily survival of both male and female An. gambiae was significantly higher in environments with sugar source densities of 5, 25, 50, 75 and 100 than density of 0 ; the daily survival was also significantly higher in environments with sugar source densities of 25, 50, 75 and 100 than in sugar source density of 5 . Difference in daily survival was not significant between sugar source densities of 25, 50, 75 and 100 . Human biting rate was only significantly different between scenarios of density 0 and density 25 and higher, between density 5 and density 100. For different densities of resting sites, daily survival was only significantly different between density 0 and other density levels, human biting rate was only significantly different between density 0 and density 20 and higher.

ANOVA of average AUCs also shows that distributions of sugar sources and outdoor resting sites at the same

Table 2 Means of daily survival rate, human biting rate and daily abundance in different scenarios

\begin{tabular}{|c|c|c|c|c|c|c|c|c|c|c|c|}
\hline \multirow[t]{2}{*}{ Scenarios } & & \multicolumn{2}{|c|}{$\begin{array}{l}\text { Male daily survival } \\
\text { rate (\%) }\end{array}$} & \multicolumn{2}{|c|}{$\begin{array}{l}\text { Female daily survival } \\
\text { rate }(\%)\end{array}$} & \multicolumn{2}{|c|}{ Human biting rate } & \multicolumn{2}{|c|}{ Male abundance } & \multicolumn{2}{|c|}{ Female abundance } \\
\hline & & Means & SD & Means & SD & Means & SD & Means & SD & Means & SD \\
\hline \multirow[t]{6}{*}{ Sugar resource density } & 0 & 2.53 & 3.67 & 2.53 & 3.67 & 0.00 & 0.00 & 0.82 & 7.60 & 0.82 & 7.60 \\
\hline & 5 & 82.37 & 10.24 & 91.95 & 8.48 & 0.26 & 0.29 & 187.44 & 120.76 & 703.38 & 411.23 \\
\hline & 25 & 84.31 & 6.97 & 93.85 & 4.47 & 0.52 & 0.78 & 349.47 & 305.27 & 1119.95 & 806.10 \\
\hline & 50 & 84.65 & 6.78 & 94.15 & 3.87 & 0.75 & 1.42 & 408.84 & 419.02 & 1265.72 & 1093.40 \\
\hline & 75 & 84.94 & 6.34 & 94.40 & 3.41 & 0.88 & 1.81 & 454.90 & 478.49 & 1372.15 & 1245.94 \\
\hline & 100 & 85.05 & 6.37 & 94.48 & 3.32 & 0.94 & 1.99 & 477.27 & 501.11 & 1427.82 & 1316.65 \\
\hline \multirow[t]{7}{*}{ Resting site density } & 0 & 77.43 & 18.36 & 86.73 & 13.65 & 0.04 & 0.07 & 62.48 & 81.15 & 256.59 & 283.14 \\
\hline & 5 & 82.47 & 9.83 & 91.73 & 7.58 & 0.21 & 0.24 & 197.06 & 140.56 & 698.35 & 431.75 \\
\hline & 10 & 83.14 & 8.78 & 92.59 & 6.87 & 0.29 & 0.36 & 236.19 & 180.27 & 822.90 & 520.47 \\
\hline & 20 & 83.77 & 8.19 & 93.31 & 6.51 & 0.41 & 0.59 & 283.85 & 222.83 & 951.47 & 616.45 \\
\hline & 30 & 84.05 & 7.60 & 93.51 & 5.95 & 0.47 & 0.68 & 316.76 & 268.01 & 1038.05 & 726.16 \\
\hline & 40 & 84.16 & 7.28 & 93.66 & 5.07 & 0.52 & 0.77 & 329.85 & 275.02 & 1069.11 & 728.00 \\
\hline & 50 & 84.31 & 6.97 & 93.85 & 4.47 & 0.52 & 0.78 & 349.47 & 305.27 & 1119.95 & 806.10 \\
\hline \multirow{2}{*}{$\begin{array}{l}\text { Closeness between } \\
\text { sugar resources } \\
\text { and resting sites }\end{array}$} & random & 83.84 & 7.81 & 93.37 & 6.41 & 0.45 & 0.67 & 295.23 & 250.10 & 983.42 & 684.06 \\
\hline & adjacent & 84.03 & 8.83 & 92.41 & 4.56 & 0.48 & 0.92 & 271.74 & 287.03 & 738.05 & 528.93 \\
\hline \multirow{2}{*}{$\begin{array}{l}\text { Closeness between } \\
\text { sugar sources and } \\
\text { houses }\end{array}$} & random & 84.31 & 6.97 & 93.85 & 4.47 & 0.52 & 0.78 & 349.47 & 305.27 & 1119.95 & 806.10 \\
\hline & adjacent & 82.65 & 9.34 & 92.31 & 7.34 & 0.27 & 0.31 & 203.42 & 129.55 & 742.89 & 411.56 \\
\hline
\end{tabular}



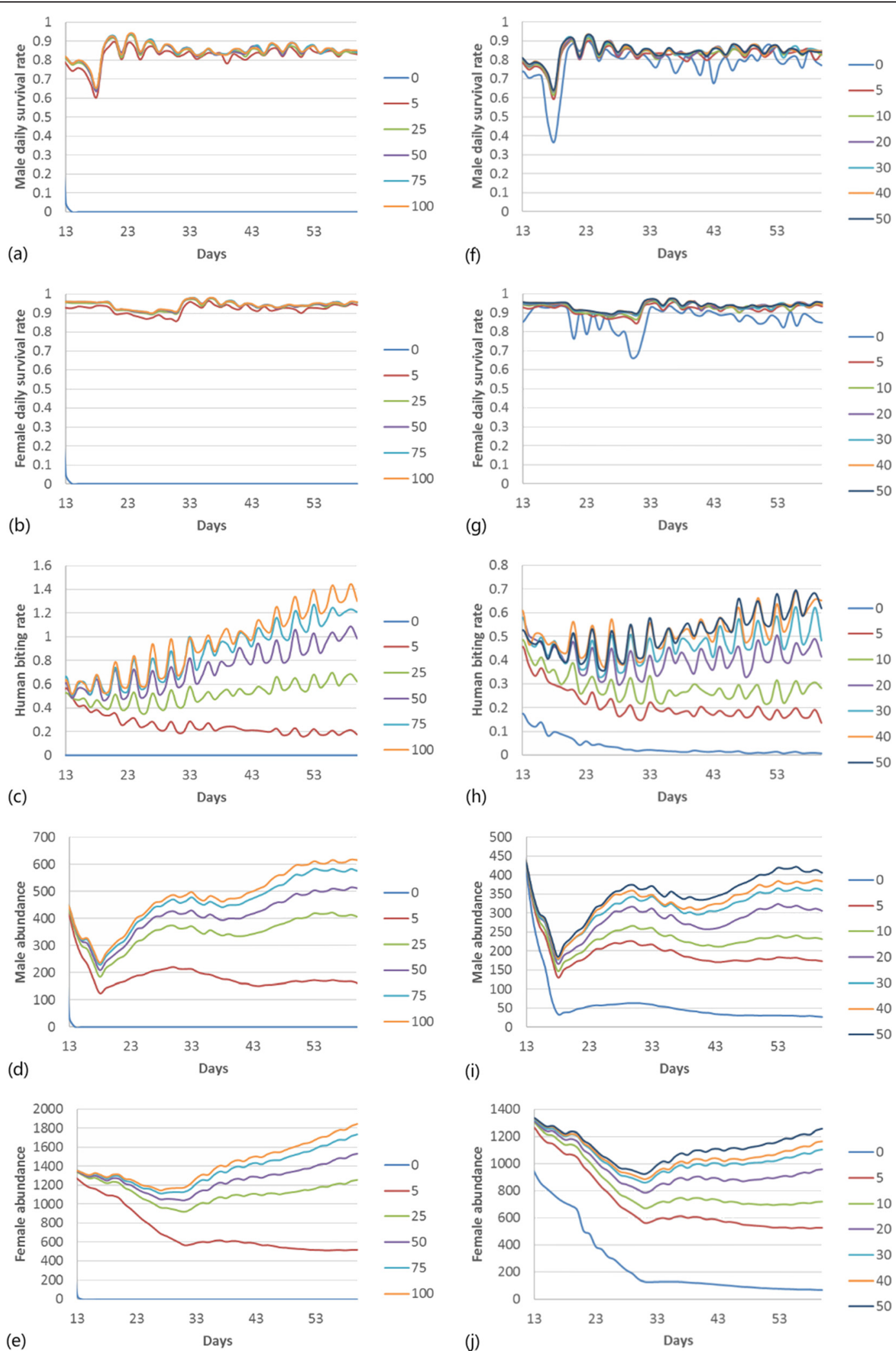

Figure 3 (See legend on next page.)
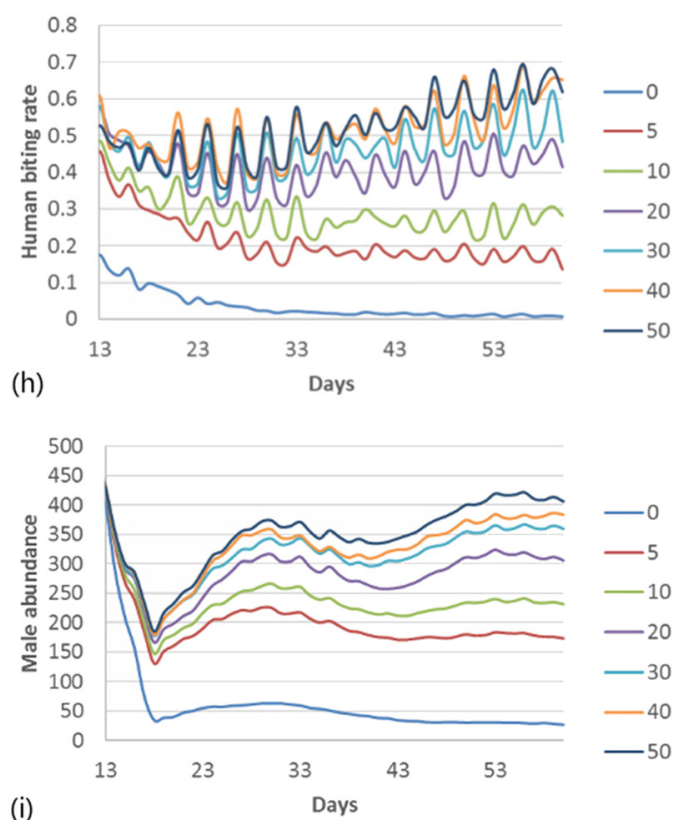

(i)

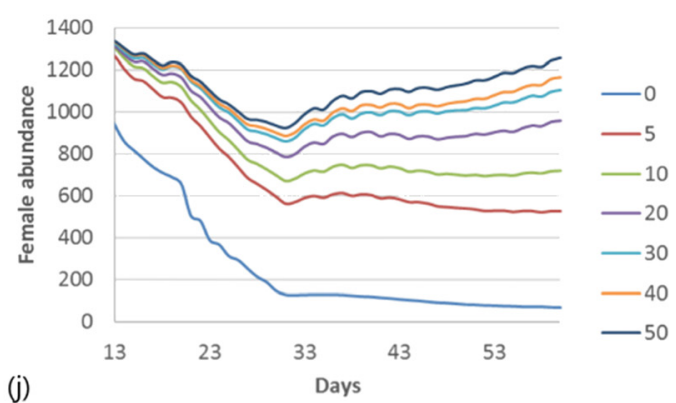


(See figure on previous page.)

Figure 3 Daily survival rates, human biting rates, and daily abundances of An. gambiae in different densities of sugar sources and outdoor resting sites. Subfigures (a) to (e) represent male daily survival rates, female daily survival rates, human biting rates, male daily abundance, and female daily abundance, respectively, of An. gambiae in environments of different sugar source densities. Subfigures (f) to (j) represent male daily survival rates, female daily survival rates, human biting rates, male daily abundance, and female daily abundance,

respectively, of An. gambiae in environments of different outdoor resting site densities. Each line represents one outcome in one density scenario.

locations or at random locations did not have a significant impact on daily survival rates, human biting rate or male abundance, but female abundance was significantly higher when sugar sources were randomly distributed in the whole village (Table 3). Having sugar sources randomly distributed in the whole village also resulted in higher survival and human biting behaviours than clustering sugar sources around houses, but only the differences of human biting rate, male and female abundance were significant (Table 3).

According to results of generalized mixed linear regression model, scenario and time were both significant factors of outcomes of daily survival rates, human biting rates and daily abundances. The interactions between scenario and time were significant factors of predicting human biting rates and abundances. After controlling the

Table 3 Comparison of average AUCs of daily survival rate, human biting rate and daily abundance in different scenarios

\begin{tabular}{|c|c|c|c|c|c|c|}
\hline Scenarios & & $\begin{array}{l}\text { Male daily } \\
\text { survival rate }\end{array}$ & $\begin{array}{l}\text { Female daily } \\
\text { survival rate }\end{array}$ & $\begin{array}{l}\text { Human biting } \\
\text { rate }\end{array}$ & $\begin{array}{l}\text { Male } \\
\text { abundance }\end{array}$ & $\begin{array}{l}\text { Female } \\
\text { abundance }\end{array}$ \\
\hline \multirow[t]{9}{*}{ Sugar resource density } & 0 & 0.0 & 0.0 & 0.0 & 19.6 & 19.6 \\
\hline & 5 & 38.2 & 42.7 & 11.9 & 8708.5 & 32869.0 \\
\hline & 25 & 39.6 & 44.1 & 24.4 & 16355.9 & 52462.2 \\
\hline & 50 & 39.8 & 44.2 & 35.0 & 19148.1 & 59313.7 \\
\hline & 75 & 39.9 & 44.4 & 41.1 & 21326.1 & 64317.8 \\
\hline & 100 & 40.0 & 44.4 & 44.3 & 22379.7 & 66938.4 \\
\hline & SE & 0.3 & 0.3 & 7.9 & 2188.5 & 5489.0 \\
\hline & $\mathrm{F}$ & 3023.89 & 3281.72 & 4.83 & 15.73 & 21.77 \\
\hline & $P$ & $<.0001$ & $<.0001$ & 0.0003 & $<.0001$ & $<.0001$ \\
\hline \multirow[t]{10}{*}{ Resting site density } & 0 & 31.0 & 38.2 & 1.7 & 2781.8 & 11814.8 \\
\hline & 5 & 38.6 & 43.0 & 9.9 & 9159.1 & 32628.5 \\
\hline & 10 & 39.1 & 43.3 & 13.5 & 11007.2 & 38490.1 \\
\hline & 20 & 39.2 & 43.6 & 19.2 & 13255.8 & 44537.5 \\
\hline & 30 & 39.5 & 43.9 & 21.8 & 14807.7 & 48612.5 \\
\hline & 40 & 39.6 & 44.0 & 24.2 & 15424.6 & 50068.3 \\
\hline & 50 & 39.6 & 44.1 & 24.4 & 16355.9 & 52462.2 \\
\hline & SE & 0.6 & 0.6 & 3.5 & 1364.5 & 3522.6 \\
\hline & $\mathrm{F}$ & 25.84 & 14.50 & 5.90 & 11.99 & 16.15 \\
\hline & $P$ & $<0.0001$ & $<.0001$ & $<.0001$ & $<.0001$ & $<.0001$ \\
\hline \multirow{5}{*}{$\begin{array}{l}\text { Closeness between sugar resources and } \\
\text { resting sites }\end{array}$} & random & 39.2096 & 43.70895 & 20.83467 & 13794.52 & 46042.25 \\
\hline & adjacent & 39.39681 & 43.41708 & 22.36233 & 12642.16 & 34496.53 \\
\hline & SE & 0.327913 & 0.324676 & 5.160015 & 1702.99 & 3545.201 \\
\hline & $\mathrm{F}$ & 0.16 & 0.4 & 0.04 & 0.23 & 5.3 \\
\hline & $P$ & 0.6873 & 0.5265 & 0.8346 & 0.6334 & 0.0234 \\
\hline \multirow{5}{*}{$\begin{array}{l}\text { Closeness between sugar sources and } \\
\text { houses }\end{array}$} & random & 39.6 & 44.1 & 24.4 & 16355.9 & 52462.2 \\
\hline & adjacent & 38.6 & 43.1 & 12.6 & 9465.6 & 34727.9 \\
\hline & SE & 0.4 & 0.4 & 3.7 & 1435.6 & 3684.0 \\
\hline & $\mathrm{F}$ & 3.59 & 2.71 & 5.07 & 11.52 & 11.59 \\
\hline & P & 0.0611 & 0.1027 & 0.0266 & 0.0010 & 0.0010 \\
\hline
\end{tabular}


effect of time, scenario was still a significant factor of affecting the outcomes (Table 4).

After controlling the effect of time (including time and its interaction with scenario in the generalized mixed linear regression model), a number of differences of the outcomes between different none-zero sugar source or outdoor resting site densities, which were not significant in ANOVA results, became significant. In addition, even when the lowest density applied in this analysis was 5 instead of 0 , the outcomes in scenarios with higher densities were still significant greater than the lowest density scenarios (Table 5).

\section{Discussion}

This study highlights how the environmental sugar sources and outdoor resting sites affect the survival and human biting rate of An. gambiae. When the numbers of sugar sources or resting sites were at low levels, small increase in their densities resulted in significant increase of daily survival rates, human biting rates, and daily abundances of An. gambiae. Time had a significant impact on the outcomes and interacted with density scenarios significantly. After eliminating the effect of time, the results show that at higher sugar source or resting site densities, increase in their densities still increased the outcomes, although the differences were less significant. Surprisingly, this model suggested that placing sugar sources at each outdoor resting site or at each house did not increase, but rather decreased survival and human biting rate of $A n$. gambiae, although the differences were only significant in three of the five outcomes.

As mentioned, further increase of sugar source and outdoor resting site density from higher density levels (sugar source density of 25 , resting site density of 10) did not increase the survival and human biting rates of An. gambiae significantly without controlling the effect of time. The reasons for this could be the following: first, the amount of increase could depend on the ratio of vector abundance and resource density. Here, only 1,000 males and 1,000 females were simulated at the beginning, so further availability of sugar sources and resting sites might have been too much and did not have an effect in this model. Second, it could be the assumptions that either sugar sources or resting sites can be reused continuously; that is, as long as the vectors could find the resource, they would be able to use it no matter how many An. gambiae mosquitoes were using them at the same time. But in reality, sugar sources like nectars are not persistent, and density of An. gambiae at certain resting sites is always limited [44-46]. So, this model may have underestimated the effect of further increase of sugar source and outdoor resting site densities, especially when real abundance of An. gambiae is high. Thus, field studies are suggested to determine the density increase of sugar sources or outdoor resting sites that can significantly impact the survival and human biting behaviour of An. gambiae in real environment. Instead of an increase of sugar source or outdoor resting site densities, it is also possible to test removing sugar sources or outdoor resting sites in field studies. Or, on the other hand, the model assumptions of initial mosquito abundances and the capability of each sugar source and resting site can be adjusted with field study results and provide more accurate predictions.

As shown in the results, a reduction in the number of sugar sources to very low levels is expected to significantly reduce mosquito numbers. This is apparently difficult in resource-rich settings, where removing certain amount of sugar sources can be meaningless; however, in resource-poor settings, further eliminating sugar sources can greatly reduce mosquito abundance, which can be used as a mosquito control strategy in sugar poor areas. Another consideration is that decreased availability of sugar source may increase blood-feeding behaviour of each female [5]. This is consistent with the results, which shows that sugar source density decrease from 25 to 5 reduced mosquito survival significantly but not the human biting rate. Thus, instead of removing all the sugar sources, placing ATSB stations near natural sugar sources or houses, or spraying ATSB solutions on vegetation may have better sugar blocking and vector control results. For resting sites, only reducing the number of outdoor resting sites to 0 had a significant effect on mosquito survival and human biting. In other words, with houses as indoor resting sites, very small number of outdoor resting sites can support mosquito survival very well. Thus in order to

Table 4 Generalized mixed linear regressions of influence of scenario and time on daily survival rates, human biting rates and daily abundances (F(df);P)

\begin{tabular}{|c|c|c|c|c|c|c|}
\hline & \multicolumn{3}{|c|}{ Sugar source density } & \multicolumn{3}{|c|}{ Resting site density } \\
\hline & Scenario & Day & Scenario $\times$ day & Scenario & Day & Scenario $\times$ day \\
\hline Male survival rate & $43.24(4) ;<0.0001$ & $148.08(47)_{i}<0.0001$ & $0.89(188) ; 0.8451$ & $1.75(5) ; 0.1225$ & $227.77(47) ;<0.0001$ & $0.91(235) ; 0.8365$ \\
\hline Female survival rate & $22.49(4) ;<0.0001$ & $46.61(47) ;<0.0001$ & 0.96(188);0.645 & $9.86(5) ;<0.0001$ & $38.37(47)_{i}<0.0001$ & $0.79(235) ; 0.9924$ \\
\hline Human biting rate & $2.85(4) ; 0.0246$ & $28.38(47) ;<0.0001$ & $1.66(188) ;<0.0001$ & $4.32(5) ; 0.0008$ & $33.58(47)_{i}<0.0001$ & $1.35(235) ; 0.0003$ \\
\hline Male abundance & $412.61(4) ;<0.0001$ & $12.03(47) ;<0.0001$ & $1.19(188) ; 0.0364$ & $4.92(5) ; 0.0002$ & $148.89(47) ;<0.0001$ & $5.45(235)_{i}<0.0001$ \\
\hline Female abundance & $3.43(4) ; 0.0083$ & $83.6(47) ;<0.0001$ & $4.09(188)_{i}<0.0001$ & $2.86(5) ; 0.0137$ & $210.74(47) ;<0.0001$ & $2.46(235)_{i}<0.0001$ \\
\hline
\end{tabular}


Table 5 Comparison of daily survival rates, human biting rates and daily abundances between different sugar source and resting site densities after controlling time $(P)$

\begin{tabular}{|c|c|c|c|c|c|c|c|c|c|c|c|}
\hline & \multicolumn{5}{|c|}{ Sugar source density } & \multicolumn{6}{|c|}{ Resting site density } \\
\hline & & s5 & s25 & s50 & s75 & & r5 & $\mathrm{r} 10$ & $\mathrm{r} 20$ & r30 & $\mathrm{r} 40$ \\
\hline \multirow[t]{5}{*}{ Male daily survival rate } & $s 25$ & $<0.0001$ & & & & $r 10$ & 0.3500 & & & & \\
\hline & $s 50$ & $<0.0001$ & 0.1661 & & & r20 & 0.1024 & 0.4830 & & & \\
\hline & s75 & $<0.0001$ & 0.0099 & 0.2271 & & r30 & 0.0364 & 0.2445 & 0.6432 & & \\
\hline & s100 & $<0.0001$ & $<0.0001$ & 0.0957 & 0.6448 & $r 40$ & 0.0255 & 0.1915 & 0.5444 & 0.8863 & \\
\hline & & & & & & r50 & 0.0149 & 0.1314 & 0.4183 & 0.7290 & 0.8388 \\
\hline \multirow[t]{5}{*}{ Female daily survival rate } & s25 & $<0.0001$ & & & & $\mathrm{r} 10$ & 0.0353 & & & & \\
\hline & $s 50$ & $<0.0001$ & 0.3772 & & & r20 & $<0.0001$ & 0.0425 & & & \\
\hline & $s 75$ & $<0.0001$ & 0.1107 & 0.4746 & & r30 & $<0.0001$ & 0.0058 & 0.4605 & & \\
\hline & s100 & $<0.0001$ & 0.0679 & 0.3434 & 0.8158 & $r 40$ & $<0.0001$ & 0.0017 & 0.2582 & 0.6937 & \\
\hline & & & & & & r50 & $<0.0001$ & 0.0003 & 0.1069 & 0.3802 & 0.6284 \\
\hline \multirow[t]{5}{*}{ Human biting rate } & s25 & 0.2635 & & & & r10 & 0.1592 & & & & \\
\hline & s50 & 0.0387 & 0.3391 & & & $r 20$ & 0.0217 & 0.1592 & & & \\
\hline & $s 75$ & 0.009 & 0.1319 & 0.5801 & & r30 & 0.0034 & 0.0409 & 0.5214 & & \\
\hline & s100 & 0.0039 & 0.0743 & 0.4049 & 0.7795 & $r 40$ & 0.0005 & 0.0086 & 0.2188 & 0.5552 & \\
\hline & & & & & & r50 & 0.0004 & 0.0078 & 0.2065 & 0.5330 & 0.9733 \\
\hline \multirow[t]{5}{*}{ Male abundance } & s25 & $<0.0001$ & & & & r10 & 0.1478 & & & & \\
\hline & s50 & $<0.0001$ & 0.0003 & & & $r 20$ & 0.0041 & 0.1555 & & & \\
\hline & $s 75$ & $<0.0001$ & $<0.0001$ & 0.0001 & & r30 & 0.0005 & 0.0435 & 0.5491 & & \\
\hline & s100 & $<0.0001$ & $<0.0001$ & $<0.0001$ & 0.1424 & $\mathrm{r} 40$ & 0.0002 & 0.0218 & 0.3825 & 0.7839 & \\
\hline & & & & & & r50 & $<0.0001$ & 0.0094 & 0.2386 & 0.5622 & 0.7601 \\
\hline \multirow[t]{5}{*}{ Female abundance } & s25 & 0.0601 & & & & $\mathrm{r} 10$ & 0.3558 & & & & \\
\hline & $s 50$ & 0.0112 & 0.5107 & & & $r 20$ & 0.0606 & 0.3405 & & & \\
\hline & s75 & 0.0025 & 0.2551 & 0.631 & & r30 & 0.0118 & 0.1107 & 0.5209 & & \\
\hline & s100 & 0.0011 & 0.1647 & 0.4645 & 0.8016 & $r 40$ & 0.006 & 0.068 & 0.3831 & 0.8179 & \\
\hline & & & & & & r50 & 0.006 & 0.0277 & 0.2117 & 0.5438 & 0.7063 \\
\hline
\end{tabular}

achieve good vector control via manipulating resting sites, disabling indoor resting sites should be emphasized.

Although it may appear that providing sugar sources where the vectors rest can make sugar seeking easier and hence improve their survival, the results indicated that it may not be the case. The explanation is that $A n$. gambiae may need sugar for energy before blood feeding or ovipositing, or for flight for other targets. Thus, sugar sources distributed over the whole village can provide a better supporting environment. This result also agrees with some of the preliminary field study observations that mosquitoes in Mali do not sugar feed near their resting sites, and there is no overlap in mosquitoes going to either resource (unpublished data).

Other IVM methods such as LLINs have not been considered in the model, so the human biting rates can be overestimated. Also, field studies providing more information can help to better adjust the model assumptions/rules, for example, the model can be improved by including the features of variation in sugar-source quality and different sugar-feeding patterns, inadequate blood meals and its effect on fecundity. With the large scale and high temporal resolution, the model can be slowed down and it will take longer to complete the simulation than the other simplified models.

While other models use parameters such as daily mortality rate $[47,48]$, which always varies in different environment conditions such as different sugar source availabilities [7], this model only uses the basic characteristics of $A n$. gambiae mosquitoes, such as their average life span, and determines the daily mortality rates by the model itself in the given scenarios, by adding the numbers of mosquito deaths due to different reasons, which is more accurate. For example, mosquitoes not able to find an energy resource for a whole night would die of starvation, mosquitoes that reached the maximum age would die of age, and the model recorded all these deaths to calculate the daily mortality rate. Also, human biting rates 
vary largely among different environmental conditions and mosquito species, while the human biting rates estimated in this model are consistent with the previously recorded range [49].

In other models with temporal resolutions of one hour or even one day, the individuals like the An. gambiae mosquitoes can only have one chance to be presented in a specific state and seek for resources or move a grid cell in every hour/day, which can underestimate their success rate of finding a resource, and devoid the capacity to express the impact of the spatial configuration. The reason for these limitations is that resources are located in the large grid cells, and mosquitoes can only find the resource if it is located in the adjacent eight grid cells. However in this model using a one-second temporal resolution, the mosquitoes can check their states, decide their next move and complete an action in every step/ second, which is more akin to real conditions. It allows the mosquitoes many chances to reach food, and can take the distance from the resources into account.

With the individual level simulation, all steps an individual mosquito or human takes can be tracked and all the details such as how many female mosquitoes died from starvation, how many mosquitoes feed on one particular sugar source, and even the route of moving of any mosquito during the whole simulation can be obtained. This capability of this model allows the development of many other hypotheses and their examination.

\section{Conclusions}

According to this model, increases in the number of sugar sources and resting sites in resource poor scenarios significantly promotes the survival of An. gambiae, increases their population, and increases the rate of human biting. This suggests methods of removing sugar sources in sugar poor areas and the use of ATSB to target the sugar feeding behaviour. To target the resting behaviour of the vectors, emphasis should be put on indoor resting sites because with the availability of indoor resting sites, very small number of outdoor resting sites can provide good support for the survival and human biting of An. gambiae. The results show that when sugar sources and outdoor resting sites are distributed over the whole village, they offer better support for $A n$. gambiae than when they are limited to certain areas, even located by each outdoor resting site or house. This observation emphasizes the importance of spatial configuration of resources in vector control. To target sugar sources or resting sites for vector control, field studies with real configurations of the environment and mosquito abundance are needed. Such studies will enable the determination of the level of density decrease in sugar sources or outdoor resting sites that can have a significant effect on mosquito population.

\section{Abbreviations}

ABM/IBM: Agent/individual-based model; AIC: Akaike information criterion; ATSB: Attractive toxic sugar bait; AUC: Area under curve; IRS: Indoor residual spraying; ITN: Insecticide-treated net; IVM: Integrated vector management; LSM: Larval source management; ODD: Overview design concepts, and details.

\section{Competing interests}

The authors declare that they have no competing interests.

\section{Authors' contributions}

LZ developed the model. WAQ, JCB, SFT, SD, YS and GCM provided consulting on vector biology assumptions in the model design. JMM checked the assumptions in the model. JWM helped with the coding. $\mathrm{LZ}$ did the simulations and collected the data. $\mathrm{LZ}$ and KLA analyzed the data. LZ wrote the first draft of the manuscript. DLD helped with the ODD protocol writing. All authors read, edited and approved the final manuscript.

\section{Acknowledgements}

We would like to thank Professor Chris Cosner, Professor Robert Cantrell, Professor Shigui Ruan and Harold Gill for the discussion on developing the model. Research reported in this publication was supported by the National Institute of Allergy And Infectious Diseases of the National Institutes of Health under Award Number R01Al100968. The content is solely the responsibility of the authors and does not necessarily represent the official views of the National Institutes of Health.

\section{Author details}

${ }^{1}$ Department of Public Health Sciences, Miller School of Medicine, University of Miami, Miami, Florida, USA. ${ }^{2}$ Department of Infectious Disease Epidemiology, MRC Centre for Outbreak Analysis and Modelling, Imperial College London, London, UK. ${ }^{3}$ USGS/Biological Resources Division and Department of Biology, University of Miami, Coral Gables, Florida, USA. ${ }^{4}$ Department of Marine Biology and Ecology, University of Miami, Miami, Florida, USA. ${ }^{5}$ Malaria Research and Training Center, Faculty of Medicine, Pharmacy and Odonto-Stomatology, University of Bamako, BP 1805 Bamako, Mali. ${ }^{6}$ Department of Microbiology and Molecular Genetics, IMRIC, Kuvin Centre for the Study of Infectious and Tropical Diseases, Faculty of Medicine, Hebrew University, Jerusalem, Israel.

Received: 24 September 2014 Accepted: 8 January 2015

Published online: 05 February 2015

\section{References}

1. Killeen GF, McKenzie FE, Foy BD, Schieffelin C, Billingsley PF, Beier JC. A simplified model for predicting malaria entomologic inoculation rates based on entomologic and parasitologic parameters relevant to control. Am J Trop Med Hyg. 2000;62:535-44.

2. Kelly-Hope LA, McKenzie FE. The multiplicity of malaria transmission: a review of entomological inoculation rate measurements and methods across sub-Saharan Africa. Malar J. 2009;8:19.

3. Foster WA. Mosquito sugar feeding and reproductive energetics. Annu Rev Entomol. 1995;40:443-74.

4. Kaufmann C, Briegel H. Flight performance of the malaria vectors Anopheles gambiae and Anopheles atroparvus. J Vector Ecol. 2004;29:140-53.

5. Beier JC. Frequent blood-feeding and restrictive sugar-feeding behavior enhance the malaria vector potential of Anopheles gambiae s/ and An. funestus (Diptera: Culicidae) in western Kenya. J Med Entomol. 1996;33:613-8.

6. Manda H, Gouagna LC, Foster WA, Jackson RR, Beier JC, Githure Jl, et al. Effect of discriminative plant-sugar feeding on the survival and fecundity of Anopheles gambiae. Malar J. 2007;6:113.

7. Gu W, Müller G, Schlein Y, Novak RJ, Beier JC. Natural plant sugar sources of Anopheles mosquitoes strongly impact malaria transmission potential. PLoS One. 2011;6:e15996.

8. Stone CM, Jackson BT, Foster WA. Effects of plant-community composition on the vectorial capacity and fitness of the malaria mosquito Anopheles gambiae. Am J Trop Med Hyg. 2012;87:727-36.

9. Stone C, Hamilton I, Foster WA. A survival and reproduction trade-off is resolved in accordance with resource availability by virgin female mosquitoes. Anim Behav. 2011;81:765-74. 
10. Stone CM, Jackson BT, Foster WA. Effects of bed net use, female size, and plant abundance on the first meal choice (blood vs sugar) of the malaria mosquito Anopheles gambiae. Malar J. 2012;11:3.

11. Straif SC, Beier JC. Effects of sugar availability on the blood-feeding behavio of Anopheles gambiae (Diptera: Culicidae). J Med Entomol. 1996;33:608-12.

12. Gary R, Foster W. Effects of available sugar on the reproductive fitness and vectorial capacity of the malaria vector Anopheles gambiae. J Med Entomol. 2001;38:22-8.

13. Rubio Palis Y, Curtis C. Biting and resting behaviour of anophelines in western Venezuela and implications for control of malaria transmission. Med Vet Entomol. 1992;6:325-34.

14. Roberts D, Alecrim W, Tavares A, Radke M. The house-frequenting host-seeking and resting behavior of Anopheles darlingi in southeastern Amazonas, Brazil. J Am Mosq Control Assoc. 1987;3:433-41.

15. Benoit JB, Lopez-Martinez G, Phillips ZP, Patrick KR, Denlinger DL. Heat shock proteins contribute to mosquito dehydration tolerance. J Insect Physiol. 2010;56:151-6

16. Ferguson HM, Dornhaus A, Beeche A, Borgemeister C, Gottlieb M, Mulla MS, et al. Ecology: a prerequisite for malaria elimination and eradication. PLOS Med. 2010;7:e1000303.

17. Gu W, Novak RJ. Agent-based modelling of mosquito foraging behaviour for malaria control. Trans R Soc Trop Med Hyg. 2009;103:1 105-12.

18. Zhou Y, Arifin S, Gentile J, Kurtz SJ, Davis GJ, Wendelberger BA. An agent-based model of the Anopheles gambiae mosquito life cycle. In: Proceedings of the 2010 summer computer simulation conference. Society for Computer Simulation International; San Diego, CA, USA; 2010. p. 201-8.

19. Arifin S, Davis GJ, Zhou Y: Modeling space in an agent-based model of malaria: comparison between non-spatial and spatial models. In: Proceedings of the 2011 Workshop on Agent-Directed Simulation. Society for Computer Simulation International, San Diego, CA, USA; 2011. P. 92-99.

20. Arifin SN, Madey GR, Collins FH. Examining the impact of larval source management and insecticide-treated nets using a spatial agent-based model of Anopheles gambiae and a landscape generator tool. Malar J. 2013;12:290.

21. Arifin S, Davis GJ, Zhou Y. A Spatial agent-based model of malaria: model verification and effects of spatial heterogeneity. Int J Agent Technol Syst. 2011:3:17-34.

22. Marshall JM, White MT, Ghani AC, Schlein Y, Muller GC, Beier JC. Quantifying the mosquito's sweet tooth: modelling the effectiveness of attractive toxic sugar baits (ATSB) for malaria vector control. Malar J. 2013;12:291.

23. Müller GC, Beier JC, Traore SF, Toure MB, Traore MM, Bah S, et al. Successful field trial of attractive toxic sugar bait (ATSB) plant-spraying methods against malaria vectors in the Anopheles gambiae complex in Mali, West Africa. Malar J. 2010;9:210

24. Ma BO, Roitberg BD. The role of resource availability and state-dependence in the foraging strategy of blood-feeding mosquitoes. Evol Ecol Res. 2008;10:1111-30.

25. Stone CM, Foster WA. Plant-sugar feeding and vectorial capacity. Ecology of Parasite-Vector Interactions. Wageningen Academic Publishers: Wageningen, the Netherlands; 2013.p. 35-79.

26. Gillies $M$, Wilkes $T$. The range of attraction of animal baits and carbon dioxide for mosquitoes. Studies in a freshwater area of West Africa. Bull Entomol Res. 1972;61:389-404.

27. Okumu FO, Killeen GF, Ogoma S, Biswaro L, Smallegange RC, Mbeyela E, et al. Development and field evaluation of a synthetic mosquito lure that is more attractive than humans. PLoS One. 2010;5:e8951.

28. Russell TL, Govella NJ, Azizi S, Drakeley CJ, Kachur SP, Killeen GF. Increased proportions of outdoor feeding among residual malaria vector populations following increased use of insecticide-treated nets in rural Tanzania. Malar J. 2011;10:80

29. Mwangangi JM, Mbogo CM, Orindi BO, Muturi EJ, Midega JT, Nzovu J, et al. Shifts in malaria vector species composition and transmission dynamics along the Kenyan coast over the past 20 years. Malar J. 2013;12:13.

30. Grimm V, Berger U, Bastiansen F, Eliassen S, Ginot V, Giske J, et al. A standard protocol for describing individual-based and agent-based models. Ecol Model. 2006;198:115-26.

31. Grimm V, Berger U, DeAngelis DL, Polhill JG, Giske J, Railsback SF. The ODD protocol: a review and first update. Ecol Model. 2010;221:2760-8.

32. Kligler I. Flight of anopheles mosquitoes. Trans R Soc Trop Med Hyg. 1924:18:199-202
33. Midega JT, Mbogo CM, Mwambi H, Wilson MD, Ojwang G, Mwangangi JM, et al. Estimating dispersal and survival of Anopheles gambiae and Anopheles funestus along the Kenyan coast by using mark-release-recapture methods. J Med Entomol. 2007:44:923.

34. Anopheles mosquitoes. [http://www.cdc.gov/malaria/about/biology/ mosquitoes/]

35. Foster W, Hancock R. Nectar-related olfactory and visual attractants for mosquitoes. J Am Mosq Control Assoc. 1994;10:288-96.

36. Gary R, Foster W. Diel timing and frequency of sugar feeding in the mosquito Anopheles gambiae, depending on sex, gonotrophic state and resource availability. Med Vet Entomol. 2006;20:308-16.

37. Charlwood JD, Pinto J, Sousa CA, Ferreira C, Petrarca V, do E Rosario V. 'A mate or a meal'-Pre-gravid behaviour of female Anopheles gambiae from the islands of São Tomé and Príncipe, West Africa. Malar J. 2003;2:9.

38. LYIMO EO, Takken W. Effects of adult body size on fecundity and the pre gravid rate of Anopheles gambiae females in Tanzania. Med Vet Entomol. 1993;7:328-32.

39. Pates H, Takken W, Stuke K, Curtis C. Differential behaviour of Anopheles gambiae sensu stricto (Diptera: Culicidae) to human and cow odours in the laboratory. Bull Entomol Res. 2001;91:289-96.

40. Knols BG, Njiru BN, Mathenge EM, Mukabana WR, Beier JC, Killeen GF. MalariaSphere: a greenhouse-enclosed simulation of a natural Anopheles gambiae (Diptera: Culicidae) ecosystem in western Kenya. Malar J. 2002;1:19.

41. Kirby M, Lindsay S. Responses of adult mosquitoes of two sibling species, Anopheles arabiensis and A. gambiae ss (Diptera: Culicidae), to high temperatures. Bull Entomol Res. 2004;94:441-8.

42. Bayoh M, Lindsay S. Effect of temperature on the development of the aquatic stages of Anopheles gambiae sensu stricto (Diptera: Culicidae). Bull Entomol Res. 2003;93:375-82.

43. Luke S, Cioffi-Revilla C, Panait L, Sullivan K, Balan G. Mason: a multiagent simulation environment. Simulation. 2005;81:517-27.

44. Lindblade KA, Walker ED, Wilson ML. Early warning of malaria epidemics in African highlands using Anopheles (Diptera: Culicidae) indoor resting density. J Med Entomol. 2000;37:664-74.

45. Githeko A, Mbogo C, Atieli F. Resting behaviour, ecology and genetics of malaria vectors in large scale agricultural areas of Western Kenya. Parassitologia. 1996;38:481-9.

46. Hobbs JH, Sexton JD, St Jean Y, Jacques JR. The biting and resting behavior of Anopheles albimanus in northern Haiti. J Am Mosq Control Assoc. 1986;2:150-3.

47. Killeen GF, Smith TA. Exploring the contributions of bed nets, cattle, insecticides and excitorepellency to malaria control: a deterministic model of mosquito host-seeking behaviour and mortality. Trans R Soc Trop Med Hyg. 2007;101:867-80.

48. Gu W, Novak RJ. Habitat-based modeling of impacts of mosquito larval interventions on entomological inoculation rates, incidence, and prevalence of malaria. Am J Trop Med Hyg. 2005;73:546-52.

49. Vittor AY, Gilman RH, Tielsch J, Glass G, Shields T, Lozano WS, et al. The effect of deforestation on the human-biting rate of Anopheles darlingi, the primary vector of falciparum malaria in the Peruvian Amazon. Am J Trop Med Hyg. 2006;74:3-11.

\section{Submit your next manuscript to BioMed Central and take full advantage of:}

- Convenient online submission

- Thorough peer review

- No space constraints or color figure charges

- Immediate publication on acceptance

- Inclusion in PubMed, CAS, Scopus and Google Scholar

- Research which is freely available for redistribution 